\title{
Logical Derivability of the Concept of Meaning, Formulation I
}

\author{
Jonathan Chimakonam Okeke, \\ Department of Philosophy, University of Calabar, Calabar, P. M. B 1115, Cross River State Nigeria
}

\begin{abstract}
Expressions, words and symbols without reference to something else which could be called their meanings are semantically helpless. But not all expressions and words refer; some even come with ambiguities and equivocations like Golden Mountain, Chimera etc., however, any symbol which does not refer could not properly be called a symbol. So because every symbol necessarily refers to something definite, it is not the case that ambiguities and equivocations would sneak into symbolic expressions. Hence, logic becomes that science which prefers symbolic or artificial or formal language to natural language. Therefore, since "meaning" or semantics is a central focus of logic together with syntax, we attempted in this work to obtain a logical derivation of it in the symbolic language of logic devoid of the ambiguities and equivocations of natural language.
\end{abstract}

Keywords- language, logic, meaning, semantics, symbol, syntax.

\section{Introduction}

Meaning can be defined as having a fixed sense or conveying a fixed idea or a fixed thought. This means that having a transitory or tentative sense does not qualify as having meaning. In philosophy of language meaning is very crucial for our words and sentences will be useless if they contain no meaning. But meaning is not something peculiar to words or sentences alone, it can in similar way be extended to symbols or whatever stands as symbol. In this same way also, we can distinguish between what is meaningful and what is meaningless since the existence of one portends the other exists. The question however is: how might we sort them out? To this day, the criteria remain the theories of meaning. And there are three of them namely:

1. Referential

2. Ideational

3. Behavioral theories of meaning

The referential theory which has more pedigree as far as solving the problem of meaning is concerned states that an expression means what it refers to or designates (Ozumba, 51). Alson (1988) as quoted in Ozumba draws a thin line between naïve and sophisticated versions of this theory. While the naïve version holds that for a statement to be meaningful, it must refer to something other than itself which depicts words as symbols standing for something other than themselves; sophisticated version holds that the meaning of a statement is to be identified with the relation between the expression and its referent, i.e., the referential connection constitutes the meaning. On the whole, the referential theory avers that the meaning of an expression is that to which it refers. The problem however, is that there are names and words which do not refer; they are sometimes described as non-denoting (Taylor, 11). Frege regards such names as problematic (63), he insists that in a language suitable for the serious purposes of science and mathematics empty names can have no legitimate employment. Such names Taylor explains must in Frege's terms be banished from the language of science and mathematics because neither the sentences which contain them nor the thoughts which those sentences express can meaningfully be evaluated for truth and falsity (12). Frege is therefore, driven to the conclusion that sentences containing names without reference are truth-valueless. Such sentences containing empty names thus introduce ambiguities and confusions in thought. For these limitations, the symbolic or artificial or formal language was invented for logic. In his Begriffsschrift(5-6)Frege states that the inadequacy of ordinary language and the ambiguities produced there from led him to the invention of symbolic language which will prevent every presuppositions and ambiguities from sneaking into our expressions. In the same way, to grasp the full measure of metalogical terms like "meaning" (without ambiguities) we have thought it imperative to formalize the scientific concept of meaning in the language of symbolic logic hence, this formal derivation of the concept of meaning.

The ideational theory of meaning on its part holds that the meaning of an expression lies in the idea it conveys. Thus, an expression which does not convey any ideas is meaningless. It is the sense of an expression which manifests as ideas in the mind that is the meaning of that expression. What this theory maintains is that all meaningful expressions are those which convey ideas.

And finally, the behavioral theory states the meaning of words and expression lies in the responses they provoke in us when we hear them. The simple version according to Ozumba (57) holds that meaning is a function of common features of situations in which expressions are uttered and responses made to those 
utterances. While the sophisticated version lends credence to human dispositions which determine the responses produced by utterances. The thin line between the two can be explained by the fact that the same expression can evoke different responses in different hearers. On the whole, this theory pins down meanings of expressions to the behavioral responses they provoke in the hearers.

It was Donald Davidson in his "Meaning and Truth" and "Theories of Meaning..." that showed how meaning and truth are related. Robert Cummins (175) in his commentary stressed that Davidson put forward the bold conjecture that meanings are satisfaction conditions and that a Tarskian theory of truth for a language is a theory of meaning for that language. This can be broken down to the following three claims:

Davidson's Conjecture

(i) A theory of meaning for $\mathrm{L}$ is a truth-conditional semantic for $\mathrm{L}$.

(ii) To know the meaning of an expression in $\mathrm{L}$ is to know a satisfaction condition for that expression.

(iii) Meanings are satisfaction conditions.

From the foregoing, the aim of this paper is to formalize the concept of meaning so as to eliminate ambiguities and confusions that arise from natural language especially where some names and words do not refer or are adjudged empty.

\section{Meaning and Symbol}

Let us now turn to symbols and address the three key definition questions which naturally arise. The first question states: are symbols the meanings of what they symbolize and at the same time their symbols? This can be reduced to the plain definition: meaning is whatever that symbolizes it. This definition is semantically closed. What this means is that the statement is self-referential in that it contains in itself the predicate "meaning" which we are using it to define. A symbol cannot be the meaning of what it symbolizes that would mean that the symbol is pointing to itself. One key condition that must be met in defining meaning is that the defining statement must not be semantically closed, in other words self-referential. Again, we know that symbol is different from what it refers to.

The second definition question says: do the symbols mean what they point to and what they point to mean the symbols? When this is broken down we have: meaning can be defined as symbols or symbols can be defined as meaning. But the two definitions do not square in well with the subject. This is because like the first, they are semantically closed. And they are in the words of Alonzo Church (751) autological mean which the subject is at the same time the predicate and the predicate at the same time the subject or that the subject and the predicate both share common properties. Also, this suffers from what I shall dub in this paper "definitional dilemma" and is therefore "semantically checked". What I mean is this; in all the known natural languages of the world, expressions move either from left to right or from right to left but never in both directions. This definition of meaning makes possible a two-directional movement. If we can define meaning as symbols, then we can also define symbols as meaning. What I mean by semantically checked is that there is no way of deciding which direction should take preeminence. The meaning of such an expression is therefore checked. Each expression makes possible the derivation of contradictory expressions. We can apply a little logic here to show this;

1. Meaning can be defined as symbol is meaningful iff it has a symbol which points at it. We can reduce this to;

2. "M" is meaningful, iff, $m$. if we decide to negate the whole of expression (1) we will have;

3. Expression (3) is not meaningful

From here we can deduced

4. "Expression (3) is not meaningful" is meaningful iff expression (3) is not meaningful.

Therefore, expression (3) is not meaningful, is meaningful. We can apply the simple rule of commutation and obtain.

5. Expression (3) is meaningful iff expression (3) is not meaningful. If we accept the second definition of meaning it commits us to (5), which can be expressed thus;

6. That meaning can be defined as symbol is meaningful if and only if meaning can be defined as symbol is not meaningful. This is called Grelling's paradox a kind of self-contradiction that arises when subject and predicate share similar properties like the definition above. So this definition does not suffice.

We now come to the third definition question. Are symbols ordinary place markers pointing towards something else which has the meaning? This can be reduced to: meaning is the fixed thought, idea or sense which an expression (symbol) refers to. One condition for defining meaning in this way which tallies with the referential theory (by far the most accepted theory of meaning) is that the predicate must not contain its subject. In other words, its substitutional instance must not be an analytic proposition e.g. 4 means 4 things. Such propositions will be semantically closed and autological. For clarity, we shall give this formalization in firstorder functional calculus (FOL): Let the set of meaningful symbols be $\mathrm{Q}$, let a symbol be $\mathrm{T}$ and let the algorithmic system be $\mathrm{L}$. Given any system $\mathrm{L}$, if $\mathrm{Q}$ is the set of formulas and $\mathrm{T}$ is a formula, then

$\mathrm{Q} \vdash \mathrm{T}$ if $(\mathrm{Q} \vdash \mathrm{T}) \supset(\mathrm{S}, \mathrm{J}, \mathrm{Z}, \ldots, \mathrm{n} / \mathrm{T})$ iff $\mathrm{L}$ is consistent. 
But of course L can only be inconsistent if Q $-\mathrm{T}$ but not satisfiable in any of S, J, Z or any other formula in that continuum. To ascertain whether $\mathrm{L}$ is consistent which is key to establishing that this third definition of meaning pans out we may have to step out of the systems of Peano Arithmetic (PA) and ZermeloFraenkel axioms of set theory which L represents. This is because the G1 and the G2 have shown that we cannot prove their consistency within them. G1 and G2 are acronyms for Gödel's first and second incompleteness theorems and they can be stated as follows:

G1: Any effectively generated theory capable of expressing elementary arithmetic cannot be both consistent and complete. In particular, for any consistent, effectively generated formal theory that proves certain basic arithmetic truths, there is an arithmetical statement that is true, but not provable in the theory (Kleene, 250; Gödel, 28).

G2: $\quad$ For any formal effectively generated theory $\mathrm{T}$ including basic arithmetical truths and also certain truths about formal provability, $\mathrm{T}$ includes a statement of its own consistency if and only if $\mathrm{T}$ is inconsistent (Feferman, 10; Gödel, 64-65).

What these especially $\mathrm{G} 2$ surmises is that if a system is consistent, its consistency cannot be proved within it otherwise it will be inconsistent. As a result of this, we shall step over to the propositional calculus to test the consistency of system L.

\section{A Logical Derivation of the Concept of Meaning}

The referential theory of meaning states that the meaning of an expression consists in what it refers to or designates. I have chosen to use this theory because it is realistic and seems to make clear sense. Now our definition shows that expression is meaningful if it refers to something else other than itself. Therefore, expressions like (1) are self-evident.

(1) An expression is meaningful if and only if, it refers to something else.

Let us take an expression D, and form a name for it by enclosing it in quotation marks; "D". Now, what is the logical relationship between $\mathrm{D}$ and (2)?

(2) "D" is meaningful.

Well, " $\mathrm{D}$ " is meaningful if, and only if, D.

Now, for our definition of meaning to be complete we shall invent a satisfactory condition and represent it with the schema (M). This is in someway similar to Tarski's material adequately condition (Semantic, 344) which he employed in his treatment of the concept of truth. What we want to show here is that for any definition of meaning to be satisfactory it must satisfy all instances of (M).

(M) "D" is meaningful if, and only if, D

We get an instance of schema (M) by inserting an actual expression for D. e.g. "4 is meaningful" if and only if, 4 is meaningful. We have earlier stated our condition - for a definition of meaning for a language $L$ to be satisfactory, it must entail every instance of $(\mathrm{M})$.

(M) " $\mathrm{D}$ " is meaningful if and only if $\mathrm{D}$

(Where $\mathrm{D}$ is an expression of $\mathrm{L}$ )

But this condition is not easily met. A satisfactory definition of meaning commits us to: (M) "D" is meaningful if and only if D

If we negate this, we would have:

(3) Expression (3) is not meaningful

(4) "expression (3) is not meaningful" is meaningful iff expression (3) is not meaningful

But: expression (3) is "expression (3) is not meaningful" since these expressions are identical, we can validly swap them in (5) (by Leibniz's law) to get a contradiction:

(5) expression (3) is meaningful iff expression (3) is not meaningful

Now, if we have a satisfactory definition of meaning, going by the criterion we set above, we are committed to (5) that is, accepting every instance of Schema (M) entails the contradictory (5). This is called the liar paradox and it is a bitter stalemate. But we have three options; we can give up some instances of Schema (M), accept calmly a contradiction or find some other way of blocking this type of inference that led us to the liar paradox. Let us go with the last option. However, what our test has proved here is that the referential theory of meaning is not satisfactory but it is possible that this is only at a propositional level where natural languages are involved. It should be noted that propositional calculus is a logic of statements chiefly of the natural languages, while the goal of our investigations in this paper is to establish whether or not mathematical symbols are meaningful the latter belonging to the artificial or formal language. Thus in following the last option, we shall be attempting to define meaning in a formal or artificial language using of course, our preferred referential theory of meaning. Let me also point out that our definition above ran into liar paradox because our natural language expressions contain semantic closure and autological properties. Let us invent an artificial language in a system we may call wimpy logic (WL) and map out its syntax for the definition of meaning. 
The syntax of WL.

1. Names: Num. sym

2. Predicates: "M" (.)

3. If $\mathrm{n}$ is a name and $\mathrm{p}$ is a predicate, $\mathrm{p}(\mathrm{n})$ is a Wff

4. If $\mathrm{A}$ is a Wff, $\sim \mathrm{A}$ is a Wff

5. If A, B are Wff, (A.B) is a Wff

6. Nothing else is a Wff

Similarly, we can map out the semantics of the definition of meaning for WL.

Axioms (Reference \& Application Axioms)

1. P applies to an object $\mathrm{O}$ iff $\mathrm{P}$ is " $\mathrm{M}$ " and $\mathrm{O}$ "M"

2. $\mathrm{O}$ is the referent of $\mathrm{n}$ iff either (i) or (ii) holds

i. $\quad \mathrm{n}$ is Num and $\mathrm{O}$ is Num (where num represents "number")

ii. $\mathrm{n}$ is Sym and O is sym (where sym represents "symbol")

Definition (Inductive Definition of Meaning WL)

1. $\quad P(n)$ is meaningful WL iff $P$ refers to the referent of $n$

2. $\quad \sim \mathrm{A}$ is meaningful WL iff $\mathrm{A}$ is not meaningful WL

3. A.B is meaningful WL iff both $A$ and $B$ are meaningful WL

Now, in deriving all, instances of the schema (M) to test whether the definition of meaning according to the referential theory of meaning for WL is semantically closed we have to look for one instance which is satisfactory. To start with can we derive an instance of (M) for "M" (NUM)?

"M" (NUM) is meaningful WL iff "M" (NUM) is not meaningful WL (D2) iff "M" does not refer to the referent of NUM (D1)

iff "M" does not refer to NUM (A2 (i))

iff it's not the case that NUM "M" (A1)

At this point it seems we have got an instance of the schema (M) which is not semantically closed or autological. The appropriate translation of "M" (NUM) in logical English is "it's not the case that NUM "M", or it is not the case that number has meaning in itself (where it is treated as a symbol). In other words it is a mere symbol or place marker pointing (referring) to the very thing it represents or which has the meaning, which is a way of saying that the subject is not contained in the predicate.

\section{Conclusion}

Adapting this great result for mathematical logic, we can surmise that the symbols refer to something else which is the meaning and that it is not the case that meaning of an expression is contained in its symbol or that the logical symbols contain ambiguities or presuppositions or are inadequate to express thoughts as is the case in the natural language theories of meaning. What we have achieved in this formulation I of the two series paper is obtaining a formal definition of meaning that is satisfactory. In doing this our result goes beyond the natural language provisions of theories of meaning such as referential, ideational and behavioral to which we referred to in the introduction of this paper. In the next formulation II, we shall take time to apply and demonstrate this at the metalogical levels of first-order logic and related systems.

\section{References}

[1] G.O. Ozumba, Introduction to philosophy of language (Ibadan: Hope Publications, 2004)

[2] K. Taylor, Truth and meaning: an introduction to the philosophy of language (Oxford: Blackwell Publishers, 1998)

[3] G. Frege, Logical investigations. P. T. Geach (Ed). (New Haven: Yale University Press,1977)

[4] G. Frege, Begriffsschrift. in from frege to gödel: A Source Book in Mathematical Logic-1879-1931. Jean van Heijenoort (Ed). (Cambridge Mass: Harvard University Press, 1969)

[5] D. Davidson, "Truth and meaning" Synthese (17) 1967, 304-23.

[6] D. Davidson, "Theories of meaning and learnable languages" Proceedings of the 1964 International Congress of Logic, Mathematics and Philosophy, 383-94.(Amsterdam: North Holland, 1965)

[7] R. Cummins, "Meaning and truth", meaning and truth: investigations in philosophical semantics (Ed). By J. K. Campbell, M, O’Rourke and D. Shier. (New York: Seven Bridges Press, 2002)

[8] A. Church, "Comparison of Russell's resolution of the semantical antinomies with that of Tarski". The Journal of Symbolic Logic, 41 (4) 1976, $747-760$.

[9] S. Kleene, Mathematical logic (New York: Wiley, 1967)

[10] K. Gödel, "On formally undecidable propositions of the principia mathematica and related systems, I." in The Undecidables: Basic Papers on Undecidable Propositions, Unsolvable Problems and Computable Functions Martin Davis (Ed). (1965), (New York: Dover Publications, 2004)

[11] K. Gödel, 'On Undecidable propositions of formal mathematical systems' The undecidables: basic papers on undecidable propositions, unsolvable problems and computable functions, Martin Davis (Ed). (1965), (New York: Dover Publications, 2004)

[12] S. Feferman, "Deciding the undecidable: wrestling with Hilbert's problems". (Text of a lecture to a general audience at Stanford University, May 13, 1994).

[13] A. Tarski. "The semantic conception of truth and the foundations of semantics". Philosophy and Phenomenological Research 4 (1944), $341-376$. 